\title{
Effect of Pressure on the Particle Size Distribution of Espresso Coffee
}

\author{
Erick Christofer I. Gonzales ${ }^{1}$, Karl Gabriel M. Lloren ${ }^{1}$, Jihan S. Al-shdifat ${ }^{2}$, Lica B. Valdez ${ }^{3}$, \\ Krizzia Rae Gines, Emmanuel V. Garcia ${ }^{1^{*}}$ \\ ${ }^{1}$ Chemistry Department, De La Salle University, Taft Avenue Manila 1004 \\ ${ }^{2}$ Brownstone Asia-Tech Inc., Mandaluyong City 1552 \\ ${ }^{3}$ Barista Coffee Academy of Asia, San Juan City 1503
}

\begin{abstract}
Studies regarding particle size distribution, particularly in espressos are surprisingly few. Particle size distribution (PSD) is a plot that displays the average diameter versus percentage volume of the particles present in a sample. With increased awareness amongst coffee drinkers, a study aiming to report the effects of different pressure on particle size distribution of espresso from four different coffee blends was conducted. The method involved pulling of espresso shots at 7, 9, and 11 bar (or atm) from different coffee blends labeled as MA, CO, AL, and MO. Laser diffraction analysis showed different particle size groupings for each set of extraction pressure. Fine particles are within 1.20 to $28.70 \mu \mathrm{m}$. The most abundant particle size is centered at $185.4 \mu \mathrm{m}$. The particle size distribution of coffee blends composed of $100 \%$ Arabica coffee (AL and MO) but sourced from different locations, showed four similar modes or size ranges centered at $3.523,13.005,28.70$, and $185.4 \mu \mathrm{m}$ regardless of the extraction pressure or the coffee source. On the other hand, the coffee blends composed of different ratios of Arabica and Robusta (MA and CO) showed different modes depending on the pressure. This indicates the potential of PSD for characterizing the purity of a coffee blend. The particle size distribution can also give insights as to the sensory attributes of the espresso coffee.
\end{abstract}

Keywords: particle size distribution; laser diffraction; arabica-robusta blend; espresso; pressure

\section{INTRODUCTION}

Coffee has been among the most traded commodities around the world. Its consumption has been increasing throughout the years with consumers shifting their focus on to flavor quality and distinctive sensory attributes (Sunarhanum et al., 2014). Since flavor is still the most important consumer parameter, coffee drinkers nowadays increasingly prefer specialty coffee over non-specialty ones. Specialty coffee is defined by its quality from farm to cup, which has been carefully handled during harvesting, processing, drying, shipping, and beverage preparation (SCAA, 2009). The flavor it imparts is found to be unique depending on species, place of origin, climate, agricultural practices, and extraction method. (Sunarharum, 2014).

Many extractions or brewing methods are available in preparing a cup of coffee. One of which is with the use of an espresso machine. According to Mestdagh et al., (2017), an espresso is a beverage that can be prepared from roast and ground coffee by means of hot water pressure applied for a short period of time, to a compact roast and ground coffee cake by a percolation machine to obtain a 
small concentrated foamy cup. The key parameters identified for this preparation method are, water to coffee ratio, particle size, compaction, pressure, and brewing time. Among these, the extraction pressure has the main influence on the acquired flavor profile of an espresso because it is also a dependent variable with respect to the permeability of coffee beds (Corrochano, 2015). A research conducted by Andueza et al., (2002) concentrated on the effect of water pressure in the physicochemical, sensory characteristics, and essential odorants of Arabica espresso. However scientific studies are still lacking to substantiate seeming impact of such key variables on cup quality.

The grinding process of roasted coffee beans are related to a particular brewing method in that an average grind size is deemed appropriate for each method. In the preparation of espresso, a certain amount of fine particles $(<100 \mu \mathrm{m})$ are needed for pressure buildup. These directly affect both permeability and flow of water (i.e. low permeability of coffee bed results to higher extraction pressures, low total flow rate, and longer extraction times, thus leading to overextraction and change in taste). For this reason, studies were done regarding the ground coffee beans, relating its particle size distribution on flavor release (Malvern, 2012), along with investigating the effects of various brewing techniques and particle size of ground coffee on sensory profiling (Fibrianto et al., 2018). We surmised that pressure during coffee processing can influence the bean fracturing dynamics, and therefore the final size distribution. However, a cursory survey of the literature reveals very limited reports focusing on the particle size distribution in espressos, hence this study.

In line with the rising demand for high-quality coffee alongside increased consumer awareness amongst coffee drinkers (Perfect Daily Grind, 2017), the aim of this work is to investigate the effects of various pressures: 7, 9, and 11 bar (or atm) in the particle size distribution of espresso obtained from four different coffee blends by means of laser diffraction analysis.

\section{METHODOLOGY}

Materials and Reagents. The grinding of coffee beans were carried out using a Rancilio Kyro $65 \mathrm{OD}$. The holder of the ground coffee was a ridged VST Inc. precision filter basket $(58 \mathrm{~mm}$ in diameter, 20-22g nominal capacity, and 30 $\mathrm{mm}$ height). Extraction process using an espresso machine, commonly termed as pulling, was done using the Milano Italy Bezzera Arcadia Display. Plastic containers for biological samples were used to contain the espresso samples. To prevent further dissolution of coffee particles, the sample containers were placed in an ice-filled chiller during the brief transport to the laboratory. The samples were then analyzed using the Beckmann Coulter LS 13320 Laser Diffraction Particle Size Analyzer equipped with Universal Liquid Module using a $1.5 \mathrm{~mL}$ liquid dropper. Distilled water was used for the rinsing and suspension of sample in the liquid module of the instrument.

Collection of Samples. Two coffee species are used in this study: Coffea canephora (Robusta) and Coffea arabica (Arabica). Four commercial variants of the coffee beans having different compositions and origins were used for the preparation the espresso shots. Coffee blend MA is 60\% Arabica and $40 \%$ Robusta in medium-dark roast. Coffee blend CO is $20 \%$ Arabica and $80 \%$ Robusta in medium dark roast. Coffee blend-AL is $100 \%$ Arabica from Ethiopia, and Coffee blend MO is also $100 \%$ Arabica but sourced from different regions in Central America. Both AL and MO blends were roasted at a light level. Twenty (20) grams of coffee grounds were collected per extraction. The ground coffee samples were then compacted in a porta filter - a component of the espresso machine that holds the ground coffee prior to and during the brewing process, and using an accessory used for compression called a tamper. Afterwards, espresso shots were pulled at various pressures of 7,9 , and $11 \mathrm{bar}$ (or atm). Extraction temperature was fixed at $95 \pm 1^{\circ} \mathrm{C}$ and weight of coffee grounds were set at $35 \mathrm{~g}$ per shot. Samples were immediately transferred to plastic containers and refrigerated. All extracted samples were prepared by a seasoned barista at the Barista and Coffee of Asia Association (BCAA).

Particle Size Distribution Analysis. Vigorous swirling of the sample was done prior to the particle size analysis, to ensure homogeneity in aqueous solution. Samples were placed in the sample holder with a use of a dropper. The following parameters were set on the LS 13320 Laser Diffraction Particle Size Analyzer: auto-rinsing for 15 seconds, debubbling for 142 seconds, measuring offset for 60 seconds, aligning for 30 seconds, and measuring background for 60 seconds. Modes or peaks are the particle sizes that pinpoint a large abundance in a sample.

Calibration of the Instrument. Glass beads with a known particle sizes and distribution were analyzed before and after the series of analyses. The data was then compared with the accepted value prescribed by the instrument. The standard procedure in calibrating the instrument was used in running the samples so as to avoid any differences in the results basing from the parameters set. 


\section{RESULTS AND DISCUSSION}

Roasted coffee beans are a mix of fibrous materials interspersed with vast array of small organic molecules that give different coffee blends their distinctive aroma and flavor. These complex composition will change depending on how the bean is fragmentised. Thus the particle size of the ground coffee should be an important parameter in maximising the release of these aroma and flavor. Particle size distribution (PSD) is a plot showing the particle size diameter and percentage volume of the particles present in the solution. Analysis of the PSD of espresso shots from different coffee blends pulled at various pressures (Figures 1-12) showed different particle size groupings for each set. The profile of the fine particles observed within 1.20 to $28.70 \mu \mathrm{m}$ range are notably different among blends and at different pressures. Generally, a steep slope is observed at $-1.0 \mu \mathrm{m}$ for MA blend (Figs 1, 5, 9) regardless of the pulling pressure as opposed to the gradual slope for the three other blends. The fine particle size indicates higher surface area and relates to the higher degree of interaction with the extracting water molecules. In theory, fine particle sizes should promote extraction of the soluble aroma and flavor from the coffee fine granules that would result to better sensory attributes. However, PSD results show that fine particles are not present in abundant quantity. The most abundant particle size was observed to center at $185.4 \mu \mathrm{m}$, which is 7 times higher in magnitude than the fine partile sizes. Pulling the espresso at 7 bar showed that the blends CO, AL and MO (Figures 2-4) afforded the abundant particle size at $185.4 \mu \mathrm{m}$. The coffee blend MA (Figure 1) pulled at 7 bar seems to be an exemption as it gave the largest particle size at 1739 $\mu \mathrm{m}$, which may be attributed to some deviations in the sample addition or dilution during the analysis. Generally, the most abundant particle size centers at $185.4 \mu \mathrm{m}$, which is also consistent when the coffee blends were pulled at higher pressures of 9 and $11 \mathrm{bar}$ (or atm) (Figures 5-12). Thus, similar abundant particle sizes centered at $185.4 \mu \mathrm{m}$ were observed regardless of the pulling pressure and coffee composition. The result is less than the reported 240 $\mu \mathrm{m}$ average abundant particle size of coffee grounds using similar kind of brewing method (Blittersforff \& Klatt (2017). Although the particle size reported by Blittersforff \& Klatt contains $25 \%$ particles that are less than $100 \mu \mathrm{m}$ in size, the abundant size obtained in this current work is notably different by $54.6 \mu \mathrm{m}$. The particle size information in ground coffee is critical since the presence of a particle size difference of at least $2 \mu \mathrm{m}$ has been reported to affect significantly the perception of sensory attributes of food (Engelen et al., 2005; Horiba, 2018). It would be interesting to evaluate the effect of having 185.4 $\mu \mathrm{m}$ abundant particle size on the sensory attributes of coffee and compare this with different particle sizes. We surmise this would significantly affect the perception of coffee sensory attributes. Since coffee is dependent on the quality of its sensory traits, the particle size should be seriously considered as among the factors in coffee preparations.

Although the abundant particle size did not change at different pulling pressures, varying the applied extraction pressures resulted to notable differences in the texture, mouthfeel, and flavor of the espresso cups. Evaluation on the taste and preference is not the focus of this study, but systematic sensory evaluation is an interesting avenue that will be explored in the future. Nevertheless, the observed differences in the texture, mouthfeel, and flavor are associated to the so-called "body" attributes of coffee, which are classified as either light, medium, or heavy to describe the thickness that a brewed coffee imparts on the surface of the tongue (Cupping Fundamentals, 2015). These differences are attributed to the extraction of some key odorants and flavor compounds such as 2-methyl butanal and 3-methyl butanal, that are usually extracted as a function of pressure (Anduenza et al., 2002).

Comparing the modes or the particle size range in the distribution (Horiba, 2012), it can be observed that the particle size distribution for the espresso shots from coffee blends composed of $100 \%$ Arabica (AL and MO) but sourced from different locations, showed four similar modes or size ranges centered at $3.523,13.005,28.70$, and $185.4 \mu \mathrm{m}$. The identical distribution range indicates that regardless of the extraction pressure or the coffee source, a pure arabica coffee affords similar PSD modes. This observation is consistent with the results of a recent study that has also observed that PSD is independent of the coffee bean origin and processing method (Uman et al., 2016). On the other hand, the coffee blends composed of different ratios of Arabica and Robusta (MA and CO) showed different modes depending on the pressure. The espresso shots of coffee blend MA, which is composed of $60 \%$ Arabica and $40 \%$ Robusta pulled at 7, 9 and 11 bars, showed PSDs with 5 (centered at 1.322, 14.940, 28.70, 185.4, and $1739 \mu \mathrm{m}$ ), 5 (centered at 1.204, 4.048, 13.61, $28.70,185.4 \mu \mathrm{m}$ ), and 4 (centered at 1.204, 4.478, 14.94, and $185.4 \mu \mathrm{m})$ modes, respectively. The coffee blend CO that is composed of $20 \%$ Arabica and $80 \%$ Robusta pulled at 7 and 9 bars showed PSDs with 3 similar modes (centered at 5.6165, 25.15 , and $185.4 \mu \mathrm{m}$ ), while those pulled at $11 \mathrm{bar}$ showed a PSD with 2 modes (centered at 26.15 and $185.4 \mu \mathrm{m}$ ). Generally for mixed blends, it indicates that at higher pressure, the number of PSD modes decrease. The differences in the number of modes 
for espresso shots obtained from mixed coffee blends can be attributed to the diferences in the ratio of the blends. Arabica and Robusta beans have been shown to exhibit different roasting behaviors, which

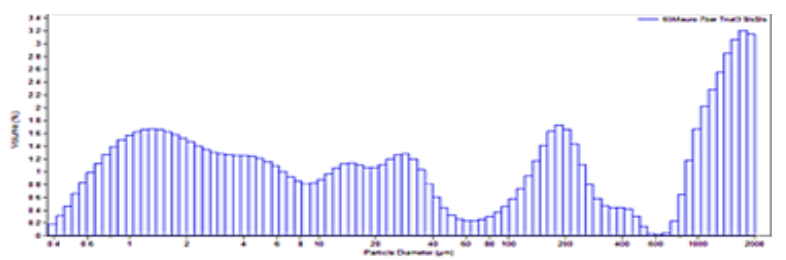

Figure 1: Particle Size vs Volume (\%) of Coffee BlendMA Pulled at 7 bar (or atm).

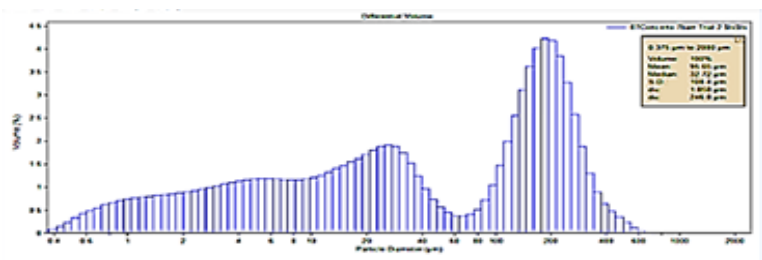

Figure 2: Particle Size vs Volume (\%) of Coffee BlendCO Pulled at 7 bar (or atm).

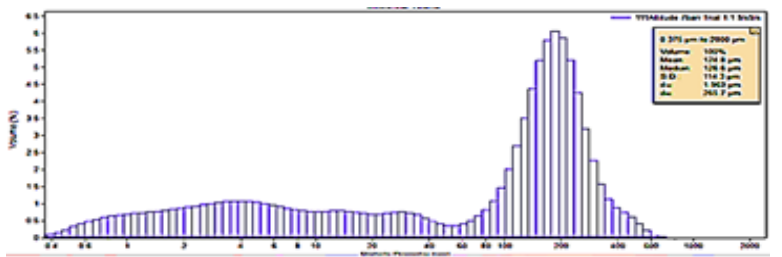

Figure 3: Particle Size vs Volume (\%) of Coffee BlendAL Pulled at 7 bar (or atm).

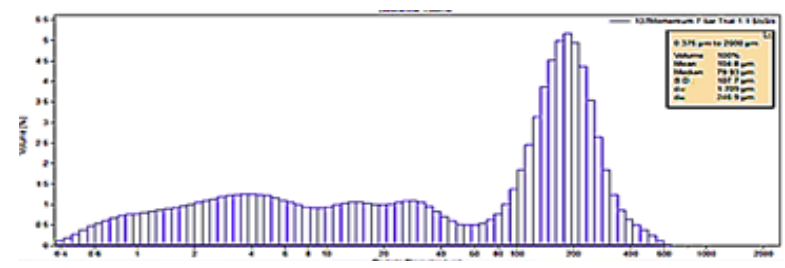

Figure 4: Particle Size vs Volume (\%) of Coffee BlendMO Pulled at 7 bar (or atm).

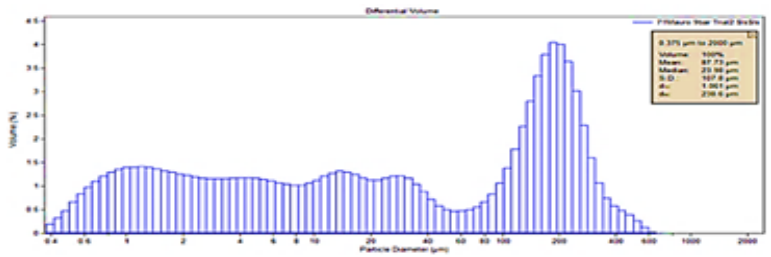

Figure 5: Particle Size vs Volume (\%) of Coffee BlendMA Pulled at 9 bar (or atm).

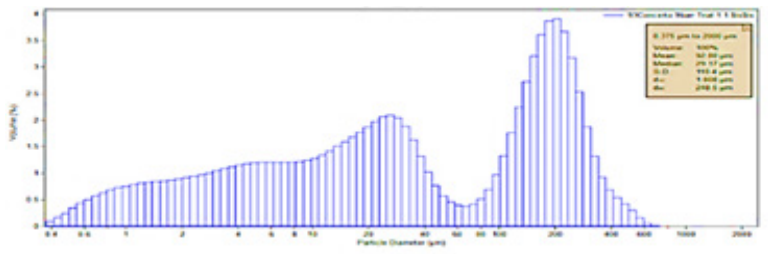

Figure 6: Particle Size vs Volume (\%) of Coffee BlendCO Pulled at 9 bar (or atm). generally affects their physical properties upon grinding. These differences have been employed by coffee roast masters in making crucial decisions whether to do blend-before-roast or blend-after-

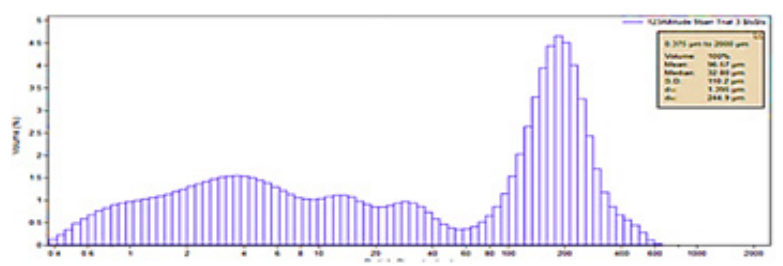

Figure 7: Particle Size vs Volume (\%) of Coffee BlendAL Pulled at 9 bar (or atm).

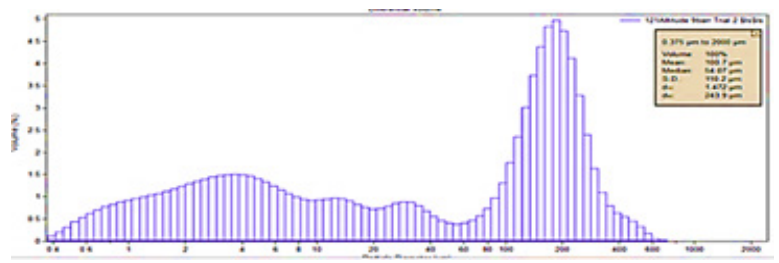

Figure 8: Particle Size vs Volume (\%) of Coffee BlendMO Pulled at 9 bar (or atm).

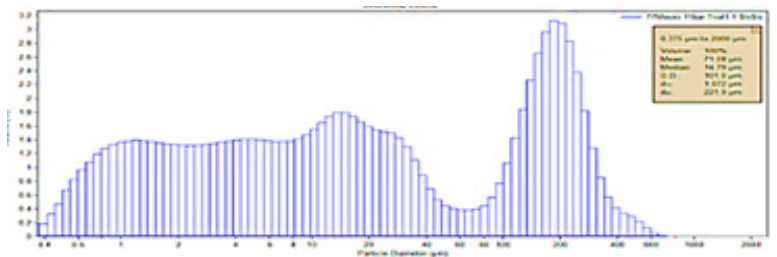

Figure 9: Particle Size vs Volume (\%) of Coffee BlendMA Pulled at 11 bar (or atm).

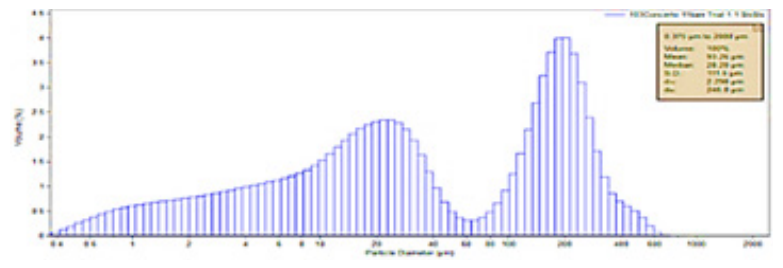

Figure 10: Particle Size vs Volume (\%) of Coffee BlendCO Pulled at 11 bar (or atm).

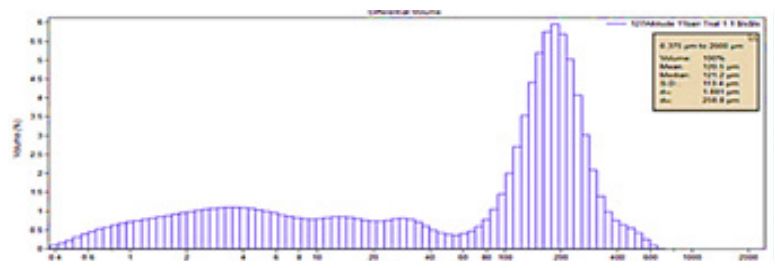

Figure 11: Particle Size vs Volume (\%) of Coffee BlendAL Pulled at 11 bar (or atm).

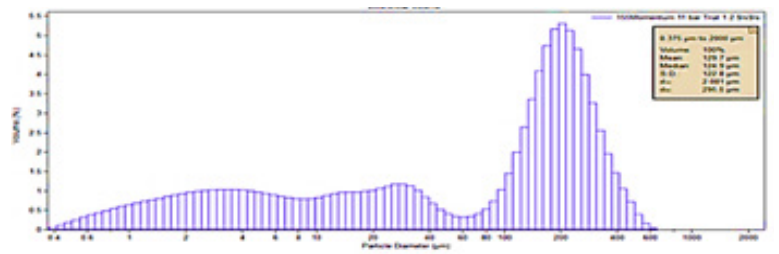

Figure 12: Particle Size vs Volume (\%) of Coffee Blend$M O$ pulled at 11 bar (or atm). 
roast during coffee preparations (Schenker \& Rothgeb, 2017).

During the process of espresso pulling, the granular material of the coffee grinds would jam the tamper especially when the pressure increases. This is a consequence of the mechanical property of granular materials (Iwashita \& Oda, 1999). Thus the variability in particle size plays a critical role especially in the flow of water. Large particles have been demonstrated by Herman (2013) to install significant order of neighboring small particles, which increases the local density resulting to the inhomogeneous flow of water. This hampered water flow affects the critical interaction of water with the coffee granules. This interaction is crucial since extraction of the soluble aroma and flavor from the coffee granule is dependent on the accessible surface area and therefore of the particle size of the ground coffee. However, the ideal particle size distribution for coffee has not been established yet and this work can shed light on the criticality of particle size. Moreover, the results indicate the potential of PSD for characterizing the purity of a coffee blend. The particle size distribution can also give insights as to the sensory attributes of the espresso coffee.

\section{CONCLUSION}

The particle size distribution of espresso extracted from different coffee blends in each set of applied pressure was notably different in fine particles within the range of 1.20 to $28.70 \mu \mathrm{m}$. Almost all of the espresso had an abundance of large particles at $185.4 \mu \mathrm{m}$ regardless of the pulling pressure. The composition of blends also showed to affect the PSD profile such that blends from 100\% Arabica displayed similar abundance of specific particle sizes, as opposed to the varying sizes observed for mixed blends of Arabica and Robusta. The distinct PSD obtained from each cup may contribute to the reason behind the unique flavor profile of espresso shots, particularly on body and mouthfeel. However correlation between size and texture perceptions using actual sensory feedbacks are still needed, along with analysis of chemical composition.

\section{ACKNOWLEDGMENTS}

The authors are thankful to the Barista and Coffee Academy of Asia (BCAA) and Brownstone AsiaTech Inc. for the help extended in the study.

\section{REFERENCES}

Andueza S, Maeztu L, Dean B, de Peña MP, Bello J, Cid, C. Influence of Water Pressure on the Final Quality of Arabica Espresso Coffee. Application of
Multivariate Analysis. J Agric Food Chem. 2002; 50(25):7426-7431.

Blittersdorff MV, Klatt C. The Grind-Particles and Particularities. In: Folmer M, ed. The Craft and Science of Coffee. 1st Ed. London:Academic Press; 2017 p. 311-328. doi:10.1016/b978-0-12-8035207.00013-x

Chemistry Coffee: Cupping Fundamentals. April 23, 2015. Retrieved on July 2018 from https://www. coffeechemistry.com/quality/cupping/cuppingfundamentals

Corrochano BR, Melrose JR, Bentley AC, Fryer PJ, Bakalis S. A new methodology to estimate the steady-state permeability of roast and ground coffee in packed beds. J. Food Eng. 2015; 150:106-116. https://doi.org/10.1016/j.jfoodeng.2014.11.006

Engelen L, de Wijk RA, van der Bilt A, Prinz JF, Janssen AM, Bosman, F. Relating particles and texture perception. Physiol Behav. 2005; 86(1-2):111-117. https://doi.org/10.1016/j. physbeh.2005.06.022

Fibrianto K, Febryana YR, Wulandari ES. Effect of brewing technique and particle size of the ground coffee on sensory profiling of brewed Dampit robusta coffee. IOP Conf. Series: Earth Envi. Sci. 2018; 131 012009. doi : 10.1088/17551315/131/1/012009

Herman A. Shear-jamming in two-dimensional granular materials with power-law grain-size distribution. Entropy 2013; 15(11): 4802-4821. https://doi.org/10.3390/e15114802

Horiba Scientific: A Guidebook to Particle Size Analysis.. 2012. Retreived on July 2018 from https:// www.horiba.com/fileadmin/uploads/Scientific/ Documents/PSA/PSA_Guidebook.pdf

Horiba Scientific: Particle Size Distribution in Flavoring and Flavorants. Retrieved on July 2018 from http://www.horiba.com/scientific/products/ particle-characterization/applications/flavoringand-flavorants/

Mastersizer 3000: Coffee Particle Size Analysis. Mastersizer Application Note MRK1884-01. Malvern Instruments Limited. 2012. Retrived on July 2018 from https://www.atascientific.com. au/wp-content/uploads/2017/02/MRK1884-01MS3K-coffee-particle-analysis.pdf

Mestdagh F, Glabasnia A, Giuliano P. The BrewExtracting for Excellence. In: Folmer M, ed. The Craft and Science of Coffee. 1st Ed. London:Academic 
Press; 2017 p. 355-380. doi:10.1016/b978-0-12803520-7.00015-3

Oda M, Iwashita K, eds. Mechanics of granular materials: an introduction. 1st ed. Rotterdam: A. A. Balkema; 1999.

Perfect Daily Grind: 59\% of US Coffee Drinkers Choose "Gourmet". 2017 March. Retrieved on July 2018 from https://www.perfectdailygrind. com/2017/03/usa-coffee-drinkers-choose-gourmet/

Rhinehart R: What is Specialty Coffee? Specialty Coffee Association of America. 2009 June. Retrieved on July 2018 from http://www.scaa. org/?page $=$ RicArtp2

Schenker S, Rothgeb T. (2017). The Roast-Creating the Beans Signature. In: Folmer M, ed. The Craft and Science of Coffee. 1st Ed. London:Academic Press; 2017 p. 245-271. doi:10.1016/b978-0-12803520-7.00011-6

Sunarharum WB, Williams DJ, Smyth HE.

Complexity of coffee flavor: A compositional and sensory perspective. Food Res Int. 2014; 62:315325. doi:10.1016/j.foodres.2014.02.030

Uman E, Colonna-Dashwood M, ColonnaDashwood L, Perger M, Klatt C, Leighton S, et al. The effect of bean origin and temperature on grinding roasted coffee. Sci Rep. 2016; 6(1): 24483. doi:10.1038/srep24483 